\title{
Update on the management of elderly patients with glioblastoma: a narrative review
}

\author{
Sarah A. Ironside ${ }^{1}$, Arjun Sahgal ${ }^{2,3}$, Jay Detsky ${ }^{2,3}$, Sunit Das ${ }^{4,5,6}$, James R. Perry ${ }^{2,7}$ \\ ${ }^{1}$ Division of Neurology, The Moncton Hospital, Dalhousie University, Halifax, Nova Scotia, Canada; ${ }^{2}$ Odette Cancer Centre, Sunnybrook Health \\ Sciences Centre, Toronto, Ontario, Canada; ${ }^{3}$ Department of Radiation Oncology, Sunnybrook Health Sciences Centre, University of Toronto, \\ Toronto, Ontario, Canada; ${ }^{4}$ Division of Neurosurgery, St. Michael's Hospital, University of Toronto, Toronto, Ontario, Canada; ${ }^{5}$ i Ka Shing \\ Institute, St. Michael's Hospital, University of Toronto, Toronto, Ontario, Canada; ${ }^{6}$ Arthur and Sonia Labatt Brain Tumour Research Centre, \\ University of Toronto, Toronto, Ontario, Canada; ${ }^{7}$ Division of Neurology, Sunnybrook Health Sciences Centre, University of Toronto, Toronto, \\ Ontario, Canada \\ Contributions: (I) Conception and design: SA Ironside; (II) Administrative support: None; (III) Provision of study materials or patients: None; (IV) \\ Collection and assembly of data: None; (V) Data analysis and interpretation: All authors; (VI) Manuscript writing: All authors; (VII) Final approval of \\ manuscript: All authors. \\ Correspondence to: Sarah A. Ironside, MD. The Moncton Hospital, 135 MacBeath Avenue, Moncton, New Brunswick, E1C 6Z8, Canada. Email: \\ Dr.Sarah.Ironside@HorizonNB.ca.
}

\begin{abstract}
Glioblastoma in the elderly (>65 years of age) is associated with shorter overall survival (OS) than in younger patients. Best practice recommendations for elderly patients, especially those with borderline or poor performance status, remain a subject of debate amongst clinicians despite recent randomized trials. This review provides an updated evidence-based summary to inform the modern management of elderly patients with glioblastoma. Based on evidence from the CE.6 randomized controlled trial, hypofractionated radiation therapy administered over a three-week course (40 Gy in 15 fractions) concomitantly with temozolomide (TMZ) followed by adjuvant TMZ has been found to be superior to radiation therapy alone with mean OS of 9.3 vs. 7.6 months and progression-free survival (PFS) of 5.3 vs. 3.9 months. This regimen should be offered to newly-diagnosed elderly patients with glioblastoma with preserved functional status, and was not associated with a negative impact on health-related quality of life (QOL). Management of elderly patients with glioblastoma can be challenging and requires a patient-centered strategy. Personalized decisions accounting for clinical, psychosocial, molecular and treatment factors are critical for realistic decision making. The importance of discussing goals-of-care with patients and their caregivers early in the disease trajectory, and establishing capacity for decision-making and advanced care planning, is also reviewed.
\end{abstract}

Keywords! Brain tumour; glioblastoma; elderly; treatment; chemoradiation; radiation; temozolomide (TMZ)

Submitted Jun 01, 2020. Accepted for publication Sep 29, 2020.

doi: 10.21037/apm-20-1206

View this article at: http://dx.doi.org/10.21037/apm-20-1206

\section{Introduction}

Glioblastoma is an aggressive and incurable primary brain cancer associated with a median overall survival (OS) of less than 15 months despite treatment, and a five year survival rate of $<10 \%$ (1). The peak incidence of glioblastoma occurs in patients 65 years of age, and advanced age is associated with an inferior survival outcome of approximately 6 months
(2-5). Elderly glioblastoma patients are more likely than younger patients to suffer from medical comorbidities and polypharmacy, to live in more precarious social situations with fewer social supports, and have reduced functional and cognitive reserve. Furthermore, toxicities from treatment are more frequent in this population. As a result, elderly patients have historically received less aggressive therapy $(6,7)$ due to concerns with respect to the risks of treatment 
Table 1 Randomized controlled trials in elderly patients with glioblastoma

\begin{tabular}{|c|c|c|c|c|c|c|}
\hline Authors & Patients & $\mathrm{N}$ & $\begin{array}{l}\text { Age cut-off } \\
\text { (years) }\end{array}$ & Intervention & Median PFS & Median OS \\
\hline Vuorinen et al. 2003 & $\begin{array}{l}\text { Radiographic evidence of } \\
\text { malignant glioma }\end{array}$ & 30 & $>65$ & Stereotactic biopsy & 72 days $^{*}$ & 85 days \\
\hline Roa et al. 2004 & Newly diagnosed GBM & 100 & $\geq 60$ & 40 Gy/15 RT & NR & 5.6 months \\
\hline \multirow{2}{*}{$\begin{array}{l}\text { Keime-Guibert et al. } \\
2007\end{array}$} & \multirow[t]{2}{*}{ Newly diagnosed GBM or AA } & \multirow[t]{2}{*}{85} & \multirow[t]{2}{*}{$\geq 70$} & 50 Gy/25 RT & 3.6 months & 7 months \\
\hline & & & & Best supportive care (BSC) & 1.5 months & 4 months \\
\hline \multirow[t]{2}{*}{ Wick et al. 2012} & \multirow[t]{2}{*}{ Newly diagnosed GBM or AA } & \multirow[t]{2}{*}{373} & \multirow[t]{2}{*}{$>65$} & 60 Gy/30 RT & 4.7 months & 9.6 months \\
\hline & & & & TMZ & 3.3 months & 8.6 month \\
\hline Malmström et al. 2012 & Newly diagnosed GBM & 291 & $>60$ & TMZ & NA & 8.3 months \\
\hline \multirow[t]{2}{*}{ Roa et al. 2015} & \multirow[t]{2}{*}{ Newly diagnosed GBM } & \multirow[t]{2}{*}{98} & \multirow[t]{2}{*}{$\geq 65$} & 40 Gy/15 RT & 4.2 months & 7.9 months \\
\hline & & & & 25 Gy/5 RT & 4.3 months & 6.4 months \\
\hline \multirow[t]{2}{*}{ Perry et al. 2017} & \multirow[t]{2}{*}{ Newly diagnosed GBM } & \multirow[t]{2}{*}{562} & \multirow[t]{2}{*}{$\geq 65$} & 40 Gy/15 RT + TMZ & 4.7 months & 9.3 months \\
\hline & & & & 40 Gy/15 RT & 3.3 months & 7.6 months \\
\hline \multirow[t]{2}{*}{ Wirsching et al. 2018} & \multirow[t]{2}{*}{ Newly diagnosed GBM } & \multirow[t]{2}{*}{75} & \multirow[t]{2}{*}{$\geq 65$} & 40 Gy/15 RT + BEV & 7.6 months & 12.1 months \\
\hline & & & & 40 Gy/15 RT & 4.8 months & 12.2 months \\
\hline
\end{tabular}

*, reported as time of deterioration, not PFS. RT, radiation therapy; TMZ, temozolomide; BEV, bevacizumab.

related toxicities and the lack of consensus with respect to a standard of care.

Historically, elderly patients have been excluded from key practice defining clinical trials, despite the fact that they represent the greater proportion of diagnosed patients. However, recent efforts to define best practice for these patients have resulted in several randomized trials (RCTs) specific to the elderly. The most recent RCT comes from the Canadian Cancer Clinical Trials Group (CCTG CE.6/EORTC). This trial contributed randomized evidence supporting the use of a 3-week hypofractionated radiation course combined with concurrent and adjuvant temozolomide (TMZ) as a standard of care for elderly patients with good performance status (8). However, for elderly patients with borderline or poor performance status, practice recommendations are inferred from the overall evidence within the context of the individual patients' needs and ability to tolerate treatment modalities (surgery, radiation, and chemotherapy). We present the following article in accordance with the NARRATIVE REVIEW reporting checklist (available at http://dx.doi.org/10.21037/ apm-20-1206).

\section{Methods}

In this narrative review, we present an up-to-date summary of key evidence to inform the management of elderly patients with glioblastoma and discuss strategies for treatment planning using a patient-centered approach. Only RCTs conducted in elderly patients with glioblastoma, published in English and identified through a MEDLINE published from 2003 to 2018 , were included and summarized in Table 1.

\section{Therapeutic interventions}

\section{Surgical resection in patients with newly-diagnosed glioblastoma}

Glioblastoma is a diffusely infiltrative tumour with a tendency to spread along axonal pathways that cannot be 
entirely removed through surgical resection. Despite this limitation, the extent of surgical resection is an important prognostic factor in glioblastoma, with a greater extent of resection associated with improved OS rates $(2,9,10)$. This finding has also been confirmed in elderly patients as reported in a small RCT of 30 elderly patients. Vuorinen et al. (11) reported a median OS of 171 days after surgical resection compared to 85 days in patients who received biopsy alone $(\mathrm{P}=0.035)$. In two recent single-institution retrospective studies $(12,13)$, the positive association between extent of resection and OS was reported only in patients who underwent surgery without experiencing post-operative complication, while the presence of a postoperative complication worsened OS in patients >age 75 . This observation highlights the increased risk of morbidity associated with neurosurgical procedures in the elderly (14).

The relationship between extent of resection and OS must be interpreted critically due to the potential for selection bias. Patients who undergo surgical resection are likely to be more medically "fit" prior to surgery, and to have tumour involving a non-eloquent potentially resectable location. In comparison, patients undergoing biopsy are more likely to have disease in an eloquent location, a factor associated with poor prognosis and shorter OS. Despite this limitation and based on the available randomized evidence, maximal safe surgical resection should be performed when feasible in appropriately selected elderly patients with newly diagnosed glioblastoma to obtain tissue for histology and to obtain molecular biomarkers that can better inform treatment decisions. Goals of surgical intervention also include tumour debulking to achieve cytoreduction, reduction of mass effect, and preservation of cognitive and functional status.

While obtaining a tissue diagnosis remains the standard of care, there remains a major need for non-invasive strategies to confirm diagnosis in elderly patients with borderline or poor performance status, in whom the risks of surgery outweigh the potential benefits. Promising non-invasive neuroimaging techniques to increase diagnostic certainty without a tissue diagnosis include hybrid techniques using amino acid PET [MET PET (15), FET PET (16)] combined with advanced multi-modal MR imaging techniques (17). Ultimately, the decision to proceed with treatment without a tissue diagnosis requires a multidisciplinary evaluation with the understanding that palliative radiation would then be the primary treatment modality as the use of chemotherapy should be directed by the molecular profile.

\section{Radiation therapy}

Radiation therapy, which can be combined with TMZ, is an integral part of the management of glioblastoma. Fractionated radiation therapy (standard dose of $60 \mathrm{~Gy}$ delivered in 30 fractions) with TMZ is the standard of care in younger patients. However, shorter hypofractionated regimens (34 Gy in 10 fractions or 40 Gy in 15 fractions) have been shown to be non-inferior (and potentially safer) than higher dose regimens in elderly patients (18-20).

ANOCEF, a French RCT, was the first to demonstrate a survival benefit in elderly patients greater than 70 years of age who received post-operative radiation therapy (50.4 Gy in 28 fractions) as compared to best supportive care alone (4). Importantly, radiation therapy did not negatively impact health-related quality of life (QOL). The RCT by Roa et al. (18) compared 6 weeks of standard radiation therapy (60 Gy in 30 fractions) to a 3-week hypofractionated course (40 Gy in 15 fractions) in elderly patients, demonstrating non-inferiority of the shorter radiation therapy course with no difference in OS. More recently, Roa et al. (19) investigated shortcourse radiation therapy (40 Gy in 15 fractions) compared to ultra-hypofractionated radiation therapy (25 Gy in 5 fractions) in elderly/frail patients with glioblastoma. No significant differences in OS, PFS or health-related QOL measures were observed between the two regimens. This is an important finding for selected patients as treatment duration is only one week and survival is extended compared to patients receiving best supportive care alone. More experience with this ultra-short course radiation course is needed as patients should be carefully selected as there can be significant risks of radiation-induced edema and necroses for larger treatment volumes.

The NORDIC trial of elderly patients (age $>60$ ) with glioblastoma (20) included three arms. Patients received either radiation alone to 60 Gy in 30 fractions, hypofractionated radiation therapy with $34 \mathrm{~Gy}$ in 10 fractions, or TMZ alone $\left(200 \mathrm{mg} / \mathrm{m}^{2}\right.$ for 5 consecutive days every 28 days for up to 6 cycles). The median OS was 1.5 months longer in the hypofractionated group as compared to the standard radiation arm, and treatment was better tolerated with fewer adverse effects ( $95 \%$ of patients in the hypofractionated group completed the full course of treatment compared to $72 \%$ in the standard radiation group). These results suggest that a hypofractionated course of radiation therapy is better tolerated and associated with at least equivalent outcomes, compared to the standard 6-week course which would otherwise be offered to younger patients. 
TMZ and other systemic therapies

TMZ is a cytotoxic oral DNA-alkylating chemotherapy, and is administered widely as the standard first line chemotherapy (combined with radiation and in the adjuvant setting) for patients with glioblastoma. For elderly patients who are unable to tolerate combined chemoradiation treatment, there is evidence to support the use of TMZ alone, however, only two clinical trials included a TMZ monotherapy arm. In a subgroup analysis of the NORDIC trial, elderly patients $>70$ years had improved OS in the TMZ arm compared to each of the two radiation therapy arms (20). In the NOA-08 trial, TMZ (1 week on and 1 week off at a $100 \mathrm{mg} / \mathrm{m}^{2}$ daily schedule) was compared to standard radiation therapy using 60 Gy delivered over 6-7 weeks (21). The median OS was not significantly different across the two treatment arms at 8.6 months in the TMZ group versus 9.6 months in the radiation therapy group $(\mathrm{P}=0.033)$. Hypermethylation of the promoter region of the gene encoding for the DNA repair enzyme $0^{6}$ - methylguanine- DNA methyltransferase (MGMT) was found to be predictive of a response to alkylating chemotherapy in both of these trials. As a biomarker, MGMT methylation status has particular clinical relevance for elderly patients with borderline performance status in whom TMZ monotherapy may be considered as a treatment option.

Of note, combined alkylating chemotherapy (TMZ/ CCNU) was administered to patients age 18-70 with MGMT promoter methylated glioblastoma in the recently published NOA-09 trial (22). A potential improvement in OS was suggested, however, this trial has a number of limitations including a small sample size and exclusion of patients $>70$ years. Therefore, the results may not be generalizable to elderly patients who are at increased risk of treatment-related toxicities.

Bevacizumab has not been found to extend the OS of elderly patients with GBM. The ARTE trial (23) compared bevacizumab combined with radiation therapy to radiation therapy alone in patients $>65$ years of age, and with a Karnofsky Performance Score (KPS) greater than or equal to 60. No significant difference in median OS between the two arms was observed (12.1 vs. 12.2 months, $\mathrm{P}=0.8$ ). However, bevacizumab was associated with a longer PFS (7.6 vs. 4.8 months, $\mathrm{P}=0.003$ ). Importantly, $52 \%$ of patients enrolled in the ARTE trial randomized to radiation therapy alone, crossed-over to receive bevacizumab upon progression which is a factor that may have contributed to the negative OS result.

\section{Concurrent chemo-radiation}

Concurrent chemo-radiation after maximal safe resection became the standard of care for newly diagnosed younger patients with glioblastoma in 2005 , as the Stupp trial demonstrated a significant survival benefit with the addition of TMZ to 60 Gy in 30 fractions of radiation therapy (24). An increase in median OS from 12.1 to 14.6 months was observed, as was an improvement in the two-year survival rate from $10 \%$ to $26 \%$. In a post hoc analysis, the survival benefit of combined treatment with TMZ + RT was smaller in older patients age 60-70 years, when compared to the benefit in patients age 50 years and younger. This raised concerns that the survival benefit was not generalizable to elderly patients (25).

With prior randomized evidence suggesting shorter radiation schedules as non-inferior and better tolerated in elderly patients $(4,18)$, and in an effort to better understand the benefit of concomitant chemoradiation in elderly patients, the NCIC CE.6/EORTC26062 trial was conducted in elderly patients ( $>65$ years; age range $65-90$ years, median age 73) and published in 2017 (8). In newly diagnosed elderly patients with glioblastoma treated with concurrent and adjuvant TMZ during short-course radiation therapy (40 Gy in 15 fractions), combined chemoradiation was found to be superior to radiation therapy alone with a median OS of 9.3 vs. 7.6 months and PFS of 5.3 vs. 3.9 months, respectively. Importantly, no significant differences were reported in health-related QOL measures between the two groups.

Analysis of patients with MGMT promoter hypermethylation in CE.6 was consistent with results from NOA-08 and the NORDIC trial, with longer OS (13.5 vs. 7.7 months) in patients with MGMT promoter hypermethylation $(\mathrm{P}<0.001)$. A clinically significant benefit of TMZ was also found in the unmethylated patients (OS 10.0 vs. 7.9 months, respectively); however, this result did not reach statistical significance $(\mathrm{P}=0.055)$. The benefit of concurrent treatment with radiation and chemotherapy in patients with unmethylated MGMT promoter was reported by Heiland et al. (13) in their recent retrospective review of elderly patients, with longer OS $(\mathrm{P}=0.009)$ in unmethylated patients receiving concurrent chemoradiation versus radiation treatment alone. At present, it remains to be determined if the addition of TMZ to RT is in fact beneficial in this molecularly defined patient cohort. As a result, if the MGMT status was known to be unmethylated at the time of a treatment decision, for elderly patients with borderline or poor performance status, we surmise that radiation alone would be considered the standard of care. 


\section{Recurrent glioblastoma}

There remains sparse evidence to guide the management of recurrent glioblastoma in the elderly. With preserved functional status, and for patients in whom the risk of treatment-related toxicity is acceptable, alternative cytotoxic chemotherapy (CCNU/lomustine) or bevacizumab can be considered at the time of tumour recurrence. Bevacizumab was approved by the FDA in the US in 2009 for recurrent glioblastoma, and is widely used for this indication. The role for tumour-treating fields (TTFields) in elderly patients requires further investigation and has significant implications for this cohort with respect to cost, QOL implications of wearing the device $>18$ hours per day, and managing the need for frequent adjustments. Lastly, at this time there is no evidence to support the use of immunotherapy or check-point inhibitors outside of clinical trials, particularly for elderly patients.

\section{Palliative care/end of life considerations}

Patients with glioblastoma experience a wide range of symptoms and can benefit from an integrative palliative care approach during the course of their illness, and at endof-life (EOL). Early introduction of palliative care with the goals of reducing physical and psychological suffering has been associated with improved QOL, longer OS (26), and can reduce unnecessary hospitalizations. EOL is a period associated with increased care requirements and clinical deterioration after anti-tumour treatments become ineffective. This period is generally confined to the three months prior to death, however, can be longer or shorter, and during this time the focus of care shifts toward symptom management and maintenance of QOL instead of prolongation of life (27).

As patients near EOL, they may lose the cognitive capacity to make decisions regarding personal care. For this reason, engaging in discussions about Advanced Care Planning (ACP) with patients and their caregivers early in the course of disease or at the beginning of the EOL phase is recommended. ACP allows the patient and their caregivers the opportunity to be actively involved in decision-making related to EOL care.

\section{Discussion}

\section{A definition of "elderly" as a construct for clinical-decision making}

Using chronological age alone to define "elderly" does not fully capture individual patient-specific factors, including physiological characteristics that determine fitness for treatment. Functional and performance status alongside cognitive reserve influence a patient's true biological or physiological age (Figure 1). Although clinicians are aware of this, for practical reasons a chronological age "cut-off" of 65 years is often selected for treatment decision-making and as inclusionary criteria in clinical trials.

\section{Determining "fitness for treatment" in elderly patients with glioblastoma}

Elderly patients with glioblastoma often experience an aggressive disease course with shortened OS as compared to younger patients, a finding that may be accounted for by treatment, tumour and "fitness" factors. These patients often present at the time of diagnosis with reduced functional status, medical comorbidities, and polypharmacy which predispose them to worse outcomes. Elderly patients are also at a higher risk of surgical complications (12), physiologic changes predisposing them to increased treatment-related toxicities, and reduced cognitive reserve predisposing them to a higher risk of neurologic toxicities. More research is needed to better understand if in addition to these factors, glioblastoma in the elderly has unique molecular features associated with more aggressive tumour biology.

In clinical practice, the most frequently used assessment tools for performance status in elderly patients with glioblastoma include the Karnofsky Performance Scale (KPS) and the Eastern Cooperative Oncology GroupPerformance Status (ECOG-PS). These assessment tools are not specifically tailored for geriatric populations and do not generally differentiate tumour-related causes from other agerelated causes of impairment. The Comprehensive Geriatric Assessment (CGA), was recently validated as a predictor of mortality in elderly patients with glioblastoma (28) and involves evaluation of neuro-cognitive, functional, nutritional and psychosocial factors; however, the CGA is both time- and resource- intensive and requires training to be properly administered. In a retrospective study of 113 elderly patients with glioblastoma intended to validate the CGA, a large number of patients were medically vulnerable; with a prevalence of frailty of $35 \%$. Standardized clinical assessments tools for vulnerable elderly neuro-oncology patients including screening measures that can predict the risk of available therapeutic interventions used alone or in combination (surgery, radiation therapy, systemic therapies) 


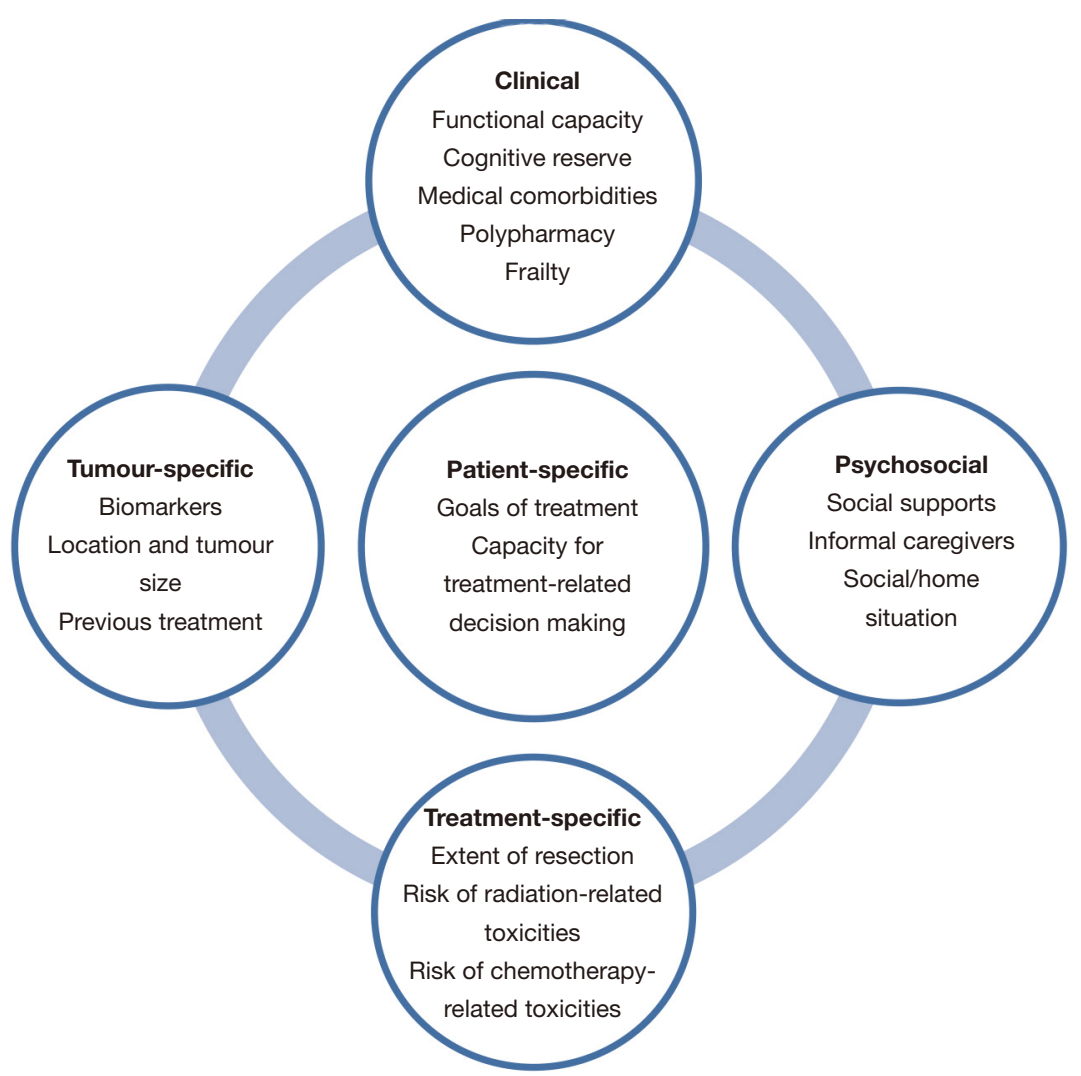

Figure 1 Treatment planning in elderly patients with glioblastoma

for individual patients are needed.

\section{MGMT promoter bypermethylation}

Across several key trials in the elderly (NOA-08, NORDIC, CE.6), methylation status of the MGMT promoter has been found to be of important prognostic and predictive significance and when this biomarker is available, MGMT should be used to inform treatment planning particularly for patients with poor or borderline performance status (Figure 2).

Wick et al. recently published the long term update of NOA-08, an RCT comparing radiation therapy to TMZ in patients greater than 65 years with anaplastic astrocytoma or glioblastoma (29). These results confirm their previous finding of MGMT promoter methylation as a prognostic and predictive biomarker. They completed an informative subgroup analysis demonstrating that the presence of MGMT promoter methylation was predictive of favourable long-term outcome in patients with one particular subtype of methylation-the RTK II subtype. This finding (in a small number of patients) suggest that patients with other methylation subclasses may not benefit from the same prognostic effect and warrants further investigation with attention to methylation profiling in elderly patients. Research to better understand the benefit of TMZ in patients with unmethylated MGMT promoter regions is urgently needed.

\section{Future directions-improving the care of elderly patients with glioblastoma}

CEC.6 established a standard for patients with KPS $\geq 70$, however a significant portion of patients present at diagnosis with borderline or poor performance status. As these patients have historically been excluded and underrepresented in clinical trials; there is a need for clinical trials in recurrent glioblastoma and in patients with borderline or poor performance status. This line of research may also lead to a better understanding of why elderly patients are less likely to receive aggressive treatment or less than the standard of care (30). The results of ongoing clinical trials 

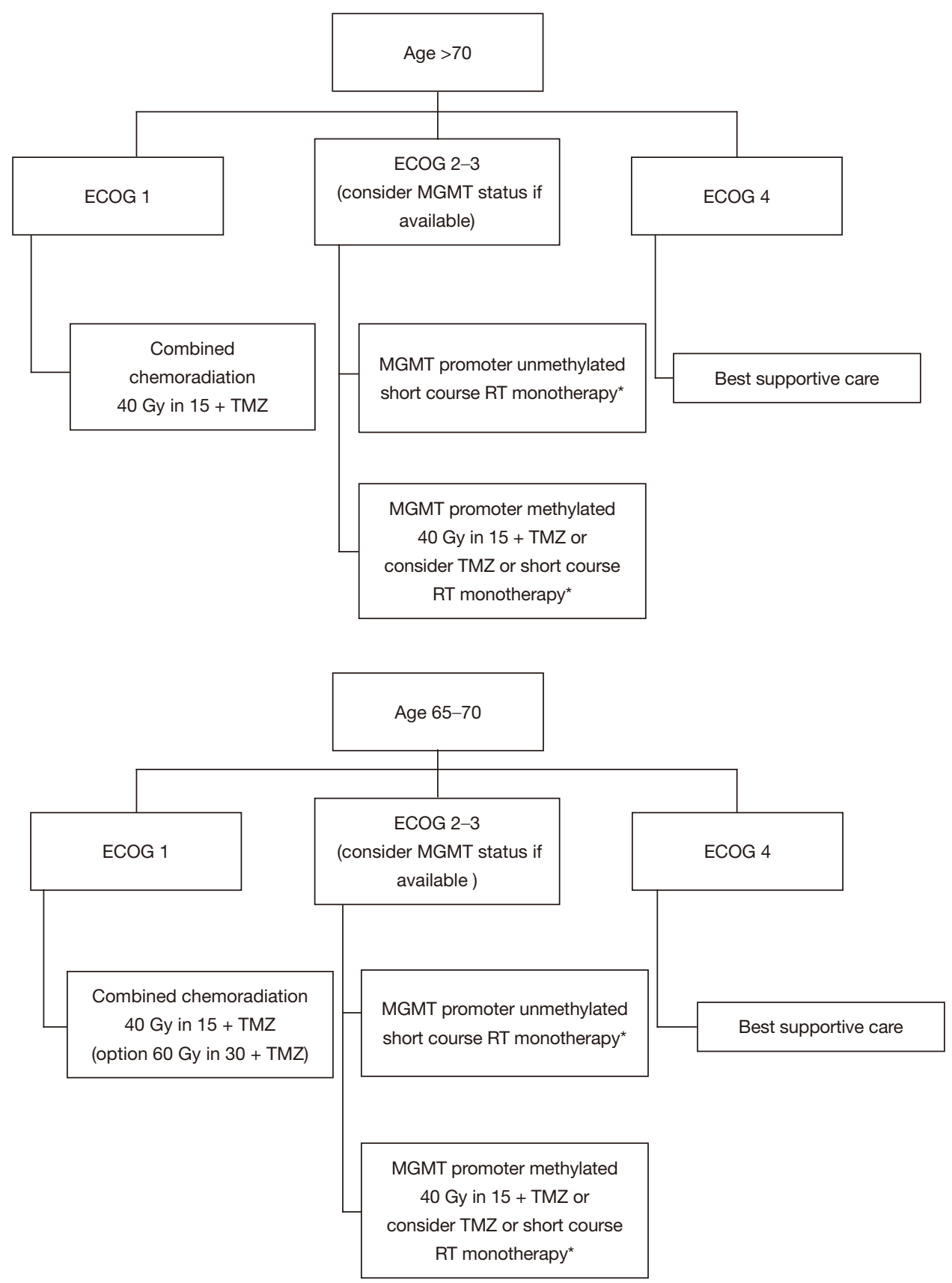

Figure 2 Treatment algorithm for newly diagnosed elderly patients with glioblastoma. *, short course RT options: 40 Gy in 15, 34 Gy in 10, 25 Gy in 5. ECOG, Eastern Cooperative Oncology Group performance status; TMZ, temozolomide; MGMT, 06-methylguanine-DNAmethyltransferase; RT, radiation therapy.

in elderly patients with glioblastoma treated with checkpoint inhibitors and small molecule cyclin-dependent kinase inhibitors alone and in combination therapies are eagerly awaited.

Dignity therapy (DT) has shown promise as a simple psychological intervention that can benefit patients with terminal illness dealing with existential and psychosocial distress to address suffering by affirming patient dignity (31). DT allows patients an opportunity through a brief psychotherapy session to discuss what matters most to 
them, to identify how they would like to be remembered and to create a legacy document to be shared with family and friends. A clinical trial is underway examining the feasibility and impact of this intervention in patients with primary brain cancer.

\section{Conclusions}

The standard of care for elderly patients with glioblastoma is redefined based on the results of CE.6. Appropriately selected elderly patients ( $\geq 65$ years), with good baseline performance status, should be offered hypofractionated radiation therapy (40 Gy in 15 fractions) combined with TMZ followed by up to 6 adjuvant cycles of maintenance TMZ. MGMT promoter methylation status (if available) can be used, based on the results of NOA-08, NORDIC and CE.6 trials to guide treatment decisions if the patient has borderline or poor performance status. In these patients, it may be appropriate to offer TMZ monotherapy, short-course radiation therapy alone, or best supportive care (Figure 2). Management of these most vulnerable patients requires a patient-centered approach, with a focus on optimizing QOL, preserving cognitive function, and minimizing treatment toxicities.

\section{Acknowledgments}

Funding: SI and JD were supported by the Gord Downie Fellowship in Brain Oncology.

\section{Footnote}

Provenance and Peer Review: This article was commissioned by the Guest Editors (Jerome Graber, Hany Soliman) for the series "Palliative Care in Neuro-Oncology" published in Annals of Palliative Medicine. The article has undergone external peer review.

Reporting Checklist: The authors have completed the NARRATIVE REVIEW reporting checklist. Available at http://dx.doi.org/10.21037/apm-20-1206

Peer Review File: Available at http://dx.doi.org/10.21037/ apm-20-1206

Conflicts of Interest: All authors have completed the ICMJE uniform disclosure form (available at http://dx.doi. org/10.21037/apm-20-1206). The series "Palliative Care in Neuro-Oncology" was commissioned by the editorial office without any funding or sponsorship. Dr. SAI reports personal fees from Hoffman-Laroche, outside the submitted work. Dr. AS reports other from Abbvie, grants and other from Elekta/Elekta AB, other from Accuray Inc., other from Varian medical systems, other from BrainLAB, other from Merck, other from Roche, other from International Stereotactic Radiosurgery Society (ISRS), other from Medtronic Kyphon, other from VieCure, outside the submitted work. Dr. SD reports grants and personal fees from Medicenna, other from Subcortical Surgery Group, grants from Alkermes, personal fees from Neinstein LLP, personal fees from McDougall Gauley, outside the submitted work. The authors have no other conflicts of interest to declare.

Ethical Statement: The authors are accountable for all aspects of the work in ensuring that questions related to the accuracy or integrity of any part of the work are appropriately investigated and resolved.

Open Access Statement: This is an Open Access article distributed in accordance with the Creative Commons Attribution-NonCommercial-NoDerivs 4.0 International License (CC BY-NC-ND 4.0), which permits the noncommercial replication and distribution of the article with the strict proviso that no changes or edits are made and the original work is properly cited (including links to both the formal publication through the relevant DOI and the license). See: https://creativecommons.org/licenses/by-nc-nd/4.0/.

\section{References}

1. Ostrom QT, Gittleman H, Truitt G, et al. CBTRUS Statistical Report: Primary Brain and Other Central Nervous System Tumors Diagnosed in the United States in 2011-2015 Neuro Oncol 2018;20:iv1-86.

2. Lacroix M, Abi-Said D, Fourney DR, et al. A multivariate analysis of 416 patients with glioblastoma multiforme: prognosis, extent of resection and survival. J Neurosurg 2001;95:190-8.

3. Brodbelt A, Greenberg D, Winters T, et al. Glioblastoma in England: 2007-2011. Eur J Cancer 2015;51:533-42.

4. Keime-Guibert F, Chinot O, Taillandier L, et al. Radiotherapy for glioblastoma in the elderly. N Engl J Med 2007;356:1527-35. 
5. Sijben AE, McIntyre JB, Roldán GB, et al. Toxicity from chemoradiotherapy in older patients with glioblastoma multiforme. J Neurooncol 2008;89:97-103.

6. Iwamoto FM, Reiner AS, Panageas KS, et al. Patterns of care in elderly glioblastoma patients. Ann Neurol 2008;64:628-34.

7. Lorimer CF, Hanna C, Saran F, et al. Challenges to treating older glioblastoma patients: the influence of clinical and tumour characteristics on survival outcomes. Clin Oncol (R Coll Radiol) 2017;29:739-47.

8. Perry JR, Laperriere N, O'Callaghan CJ, et al. Shortcourse radiation plus temozolomide in elderly patients with glioblastoma. N Engl J Med 2017;376:1027-37.

9. Simpson JR, Horton J, Scott C, et al. Influence of location and extent of surgical resection on survival of patients with glioblastoma multiforme: results of three consecutive Radiation Therapy Oncology Group (RTOG) clinical trials. Int J Radiat Oncol Biol Phys. 1993;26:239-44.

10. Brown TJ, Brennan MC, Li M, et al. Association of the Extent of Resection With Survival in Glioblastoma: A Systematic Review and Meta-analysis. JAMA Oncol 2016;2:1460-9.

11. Vuorinen V, Hinkka S, Färkkilä $M$, et al. Debulking or biopsy of malignant glioma in elderly people - a randomised study. Acta Neurochirurgica 2003;145:5-10.

12. Karsy M, Yoon N, Boettcher L, et al. Surgical treatment of glioblastoma in the elderly: the impact of complications. J Neurooncol 2018;138:123-32.

13. Heiland DH, Haaker G, Watzlawick R, et al. One decade of glioblastoma multiforme surgery in 342 elderly patients: what have we learned? J Neurooncol 2018;140:385-91.

14. Chibbaro S, Di Rocco F, Makiese O, et al. Neurosurgery and elderly: analysis through the years. Neurosurg Rev 2010;34:229-34.

15. Zhao C, Zhang Y, Wang J. A meta-analysis on the diagnostic performance of (18)F-FDG and (11) C-methionine PET for differentiating brain tumors. AJNR Am J Neuroradiol 2014;35:1058-65.

16. Dunet V, Rossier C, Buck A, et al. Performance of 18F-fluoroethyl-tyrosine (18F-FET) PET for the differential diagnosis of primary brain tumor: a systematic review and metaanalysis. J Nucl Med 2012;53:207-14.

17. Lohmann P, Werner JM, Shah NJ, et al. Combined amino acid positron emission tomography and advanced magnetic resonance imaging in glioma patients. Cancers (Basel) 2019;11:153

18. Roa W, Brasher PM, Bauman G, et al. Abbreviated course of radiation therapy in older patients with glioblastoma multiforme: a prospective randomized clinical trial. J Clin Oncol 2004;22:1583-8.

19. Roa W, Kepka L, Kumar N, et al. International Atomic Energy Agency randomized phase III study of radiation therapy in elderly and/or frail patients with newly diagnosed glioblastoma multiforme. J Clin Oncol 2015;33:4145-50.

20. Malmström A, Grønberg BH, Marosi C, et al. Temozolomide versus standard 6-week radiotherapy versus hypofractionated radiotherapy in patients older than 60 years with glioblastoma: the Nordic randomised, phase 3 trial. Lancet. Oncology 2012;13:916-26.

21. Wick W, Platten M, Meisner C, et al. Temozolomide chemotherapy alone versus radiotherapy alone for malignant astrocytoma in the elderly: the NOA-08 randomised, phase 3 trial. Lancet Oncol 2012;13:707-15.

22. Herrlinger $\mathrm{U}$, Tzaridis $\mathrm{T}, \mathrm{Mack} F$, et al. Lomustinetemozolomide combination therapy versus standard temozolomide therapy in patients with newly diagnosed glioblastoma with methylated MGMT promoter (CeTeG/ NOA-09): a randomised, open-label, phase 3 trial. Lancet 2019;393:678-88.

23. Wirsching HG, Tabatabai G, Roelcke U, et al. Bevacizumab plus hypofractionated radiotherapy versus radiotherapy alone in elderly patients with glioblastoma: the randomized, open-label, Phase II ARTE trial. Ann Oncol 2018;29:1423-30.

24. Stupp R, Mason WP, van den Bent MJ, et al. Radiotherapy plus concomitant and adjuvant temozolomide for glioblastoma. N Engl J Med 2005;352:987-96.

25. Laperriere N, Weller M, Stupp R, et al. Optimal management of elderly patients with glioblastoma. Cancer Treat Rev 2013;39:350-7.

26. Ferris FD, Bruera E, Cherny N, et al. Palliative cancer care a decade later: accomplishments, the need, next steps -- from the American Society of Clinical Oncology. J Clin Oncol 2009;27:3052-8.

27. Koekkoek JA, Chang S, Taphoorn MJ. Palliative care at the end-of-life in glioma patients. Handb Clin Neurol 2016;134:315-26.

28. Lombardi G, Bergo E, Caccese M, et al. Validation of the Comprehensive Geriatric Assessment as a Predictor of Mortality in Elderly Glioblastoma Patients. Cancers (Basel) 2019;11:1509.

29. Wick A, Kessler T, Platten M, et al. Superiority of temozolomide over radiotherapy for elderly patients with 
RTK II methylation class, MGMT promoter-methylated malignant astrocytoma. Neuro Oncol 2020;22:1162-72.

30. Dressler EV, Liu M, Garcia CR, et al. Patterns and disparities of care in glioblastoma. Neurooncol Pract
Cite this article as: Ironside SA, Sahgal A, Detsky J, Das S, Perry JR. Update on the management of elderly patients with glioblastoma: a narrative review. Ann Palliat Med 2021;10(1):899908. doi: 10.21037/apm-20-1206
2019;6:37-46.

31. Chochinov HM. Dignity-conserving care- a new model for palliative care: helping the patient feel valued. JAMA 2002;287:2253-60. 